\section{In search of our place in the Universe}

\section{Joseph Silk}

PHILOSOPHERS arise! Cosmology is beckoning. For mankind has found a niche in the Universe as it expands from an origin shrouded in mystery to an unknown future. Sir Bernard Lovell's new book tells the story of the development of human awareness of the Universe. Lovell leads us from the geocentric cosmology of the ancient world via the heliocentric cosmology of the Renaissance and the egocentric cosmology of the nineteenth century. His destination, of course, is the big bang theory of the expanding Universe, where fundamental issues still await resolution. Some of these issues differ little from the questions which faced the cosmologists of antiquity. Sir Bernard Lovell is an eminent radioastronomer who has no cosmological axe to grind, and it is especially refreshing to discover that his popular account of the evolution of cosmology manages to be both readable and enthralling.

We learn about the epicycles of Hipparchus and Ptolemy, and about how Thomas Aquinas incorporated Aristotle's crystal sphere cosmology into an apparently irrefutable argument for the existence of God. With Copernicus, the heliocentric hypothesis re-emerged and, against all odds, survived, while Tycho Brahe established the first accurate and systematic records of planetary data. Brahe punctured the immutable crystal spheres with his studies of comets and of the famous supernova of 1572 . His assistant, Johannes Kepler, derived the laws of planetary motion after a painful decade of sifting endless mathematical permutations of the planetary data, and despite unflinching belief in the celestial harmony exhibited by the five regular solids. Next, Galileo's observations with a $4 \mathrm{~cm}$ aperture telescope caused an immense stir that led to an unavoidable conflict with the geocentric dogma upheld by theologians. Galileo saw sunspots, the moons of Jupiter and the phases of Venus, all of which established that the heliocentric system was a reality, and could no longer be thought of as a computationally convenient hypothesis. It remained for Isaac Newton to express the universal law of gravitation in a way that gave a theoretical understanding of Kepler's Laws.

The stage was set for modern astronomy. One of the great pioneers was William Herschel, who counted stars and mapped our Milky Way galaxy. Not, however, until the twentieth century was the Sun finally displaced from the centre of the Universe. Harlow Shapley resoundingly overthrew the egocentric view of the place of mankind in the Universe by using the newly calibrated period-luminosity relation for Cepheid variable stars to establish distances to remote globular star clusters. These were found to surround the
Emerging Cosmology. By Bernard Lovell. Pp.208. ISBN 0-231-05304-5. (Columbia University Press: 1981.) \$14.95, $£ 10.80$.

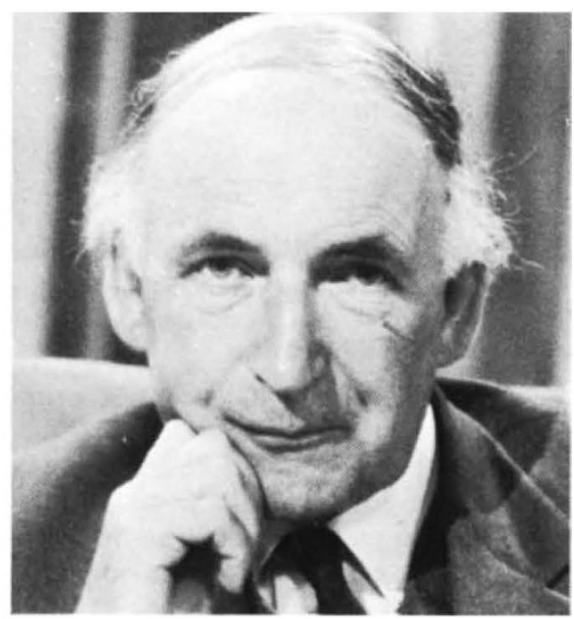

Sir Bernard Lovell, now tackling the history and philosophy of cosmology.

centre of our galaxy some 30,000 light years from the Sun. Much happened subsequently: the discoveries of the expansion of the Universe and of the cosmic microwave background radiation are two of the highlights that have now led cosmologists almost unanimously to adopt the big bang cosmological model.

All of this forms a backdrop to Lovell's theme, which is the evolution of cosmological thinking. Lovell's book forms part of a philosophical series entitled "Convergence" and founded, planned and edited by Ruth Nanda Anshen in order to explore the new consciousness which marks mankind's recently acquired ability to tinker with the evolutionary processes of nature. Our unfolding knowledge of the cosmos traditionally places cosmologists in the role of passive observers acquiescing in the thrill of new astronomical explorations of the frontiers of the Universe. Unlike most natural scientists, astronomers cannot perform controlled experiments in a warm laboratory; rather they take whatever the Universe has to offer.

One might think that such a discipline would have little in common with philosophers who concern themselves with the interaction between human beings and their environment. However a current cosmological theme provides a remarkable bridge between the philosophical undertones associated with the origin and fate of the Universe and the human perspective. This is the anthropic principle, according to which the properties of the Universe are determined by our presence as observers. Conditions must be congenial for intelligent life to evolve. This means, for example, that the early Universe could not have been highly irregular, inhomogeneous or chaotic, nor however could it have been perfectly regular, otherwise galaxies and stars would not have formed. The anthropic principle accounts for the various large-number coincidences involving powers of $10^{40}$, the ratio of electromagnetic to gravitational coupling constants. These include the mass of a star and the mass of the observable Universe, as well as the coincidence between nuclear, stellar and Hubble expansion time-scales. It even purports to account for the approximate values of the constants of nature: if they differed significantly from observed values, human beings could not have evolved.

Where does this leave us? The uniqueness of our Universe can be attributed to our existence as observers, if we accept the anthropic principle. But have we really advanced our understanding of any aspects of cosmology? In 1925, A.N. Whitehead perceived about cosmology that "there is no parting from your own shadow". Is the Universe merely our shadow? Or perhaps the cosmologists are deceiving themselves and the roles are reversed. The truth may very well be that with only one Universe to explore, we can never resolve this paradox.

Joseph Silk is Professor of Astronomy at the University of California, Berkeley, and author of The Big Bang (W.H. Freeman, 1980).

\section{All around our star}

\section{David W. Hughes}

The New Solar System. Edited by J. Kelly Beatty, Brian O'Leary and Andrew Chaikin. Pp.224. ISBN 0-521-23881-1. (Cambridge University Press: 1981.) £9.95, \$19.95. Orbiting the Sun: Planets and Satellites of the Solar System. By Fred L. Whipple. Pp.338. ISBN 0-674-64125-6. (Harvard University Press: 1981.)\$20, £14. Daytime Star: The Story of Our Sun. By Simon Mitton. Pp.191. ISBN US 0-68416840-5; ISBN UK 0-571-11659-0. (Charles Scribner's Sons/Faber \& Faber: 1981.) $\$ 14.95, £ 10$.

WE LIVE in the Solar System. Our planet, Earth, orbits the Sun and the theme of these three books seems to be that the more we know of such bodies the better. In effect we are presented with a progress report. Governments have spent large amounts of money on investigating our neighbouring planets and star. What have we got from it? Where do we go next? Why bother doing more? The books under review try to answer these questions.

The investigation of the Solar System has passed through three phases. The classical period encompassed the works of scientists such as Galileo, Copernicus, Kepler and Newton. This was a time of discovery and astronomy was almost entirely concerned with the planets, stars being 
fixed points of light of little known significance. The second phase started in the late 1800 s when astrophotography and spectroscopy spawned the discipline of astrophysics. During this period planetary investigation degenerated into a Cinderella science. The early 1960 s saw the dawn of planetology. Since then spacecraft have been flung round, onto and into all of the planets known to the ancients, producing a veritable explosion of knowledge.

The first book under review is The New Solar System. Very rarely does one come across such an excellent account; I feel honoured to have a first edition as it is bound to run to many printings. The editors have collected together 20 chapters, each of about ten pages, written by a roll call of American scientists which reads like a Who's Who of US planetology. It is invidious to omit names, but as an indication of the standard it includes Eddy on the Sun, Chapman on asteroids, Burns on planetary rings, Wood on meteorites, Masursky on Mars, Shoemaker on the role of fragmentation and so on. The book concentrates on the comparative approach. It is much easier to discuss the surfaces of the terrestrial planets (Mercury, Venus, Earth and Mars) in one chapter and, for example, their atmospheres in another than to have an isolationist attitude to individual planets.

The joy of reading the book is greatly enhanced by the illustrations. Not only does it bulge with space photographs, but it also contains a collection of coloured illustrations by artists Charles Wheeler, Don Davies and Jon Lomberg.

The editor's aim was to provide a book about the third phase of planetary exploration, the space-age planets, a book which would make enjoyable reading for those with either a professional or a casual interest. They have succeeded admirably. The fact that the publishers have produced this large format, beautifully illustrated, quality book at less than $£ 10$ is verging on the miraculous. I advise all readers to buy it.

The second book is Fred Whipple's Orbiting the Sun: Planets and Satellites of the Solar System. This is a completely revised and updated edition of Whipple's classic text, Earth, Moon and Planets, which was first published in 1941. Whipple is Philips Professor of Astronomy, Emeritus, at Harvard University and Senior Scientist, Emeritus, at the Smithsonian Institution Astrophysical Observatory. He has devoted his life to the study of astronomy and space science, especially meteors, comets, space exploration planning, satellite tracking, novae and supernovae, Solar System evolution and practical astronomy; it is fascinating to read the view of such a man about the place of Earth and Moon within the planetary context. Whipple writes for the general reader and mathematics are avoided; however, the book does present the basic foundation of planetary astronomy and

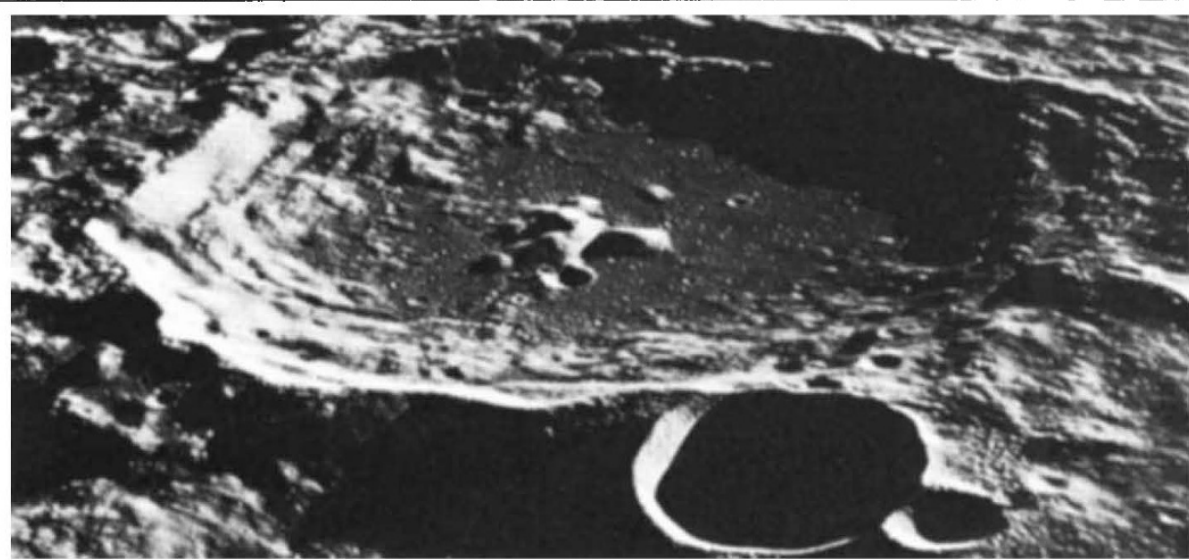

The crater Daedalus on the far side of the Moon, photographed by Armstrong and Aldrin on July 21, 1969. The picture is taken from The Atlas of the Universe by Patrick Moore, a new edition of which has just been published by Mitchell Beazley, price $£ 19.95$.

does stress the underlying scientific principles. The author has also managed to integrate the three phases of Solar System investigation and we realize that all still have a role to play. We might move from bleary telescope images to the eye-opening, crisp detail of the space probe, but the Earth-based telescopes have recently revealed Pluto's moon, the rings of Uranus and Apollo asteroids. Also we realize that stars and planets are not to be compartmentalized. Whipple leaves us in no doubt that the origins and deaths of stars are intricately connected with the origin and evolution of our planetary system. The book is well written, well illustrated, well worth reading and a worthy successor to the previous editions.

My final book is Daytime Star: the Story of Our Sun by Simon Mitton. The word "Story" in the title describes the book well - Mitton dives into the subject and returns with a net bulging with intrigues. Why did ancient man worship the Sun and build stone circles to track its path? What drives the sunspots? Does the solar energy output vary slightly and thus trigger ice ages? What do neutrinos tell us about the solar interior? What have we learnt from space about the solar atmosphere? Mitton has a racy style and we find atomic particles sloshing, neutrons bashing, gases toasting and gravity crunching.

There is much to be learnt about the Sun from this book, and it fills a definite gap in the astronomical library of the general reader. The author worried me when he stated that in 1964 "the finding of the solar wind was a result of astronomical observations of extremely remote radio sources", but 86 pages later he changes his mind and Biermann's 1951-1953 observations of comet tail phenomena are given the credit. I also found that the position of the Sun in the stellar hierarchy needed much more emphasis and that it is not enough just to say that the Sun has "an average size, typical mass, normal structure, temperature and luminosity". Granted the Sun is on the Main Sequence but it is also about nine times more massive than the average star and about 2,000 times more luminous. But these are minor quibbles about a book which is generally of a high standard and is definitely a good read.

David $W$. Hughes is a Lecturer in Astronomy and Physics at the University of Sheffield.

\section{The philosophy of astronomical discovery}

\section{David S. Evans}

Cosmic Discovery: The Search, Scope, and Heritage of Astronomy. By Martin Harwit. Pp.334. ISBN UK 0-7108-0089-4; ISBN US 0-465-01428-3. (Harvester Press, Brighton/Basic Books, New York: 1981.) $£ 12.95, \$ 25$.

IN the blurb Sir Fred Hoyle describes Cosmic Discovery as "A remarkable book. A unique book". So it is. It is an examination of the epistemology of astronomy, and one of those books which will set astronomers and philosophers thinking and talking. It is not a book which will command general acquiescence. Exactly how much influence it will have is hard to say since it is certainly not everybody's cup of tea.

It starts off, oddly enough, with an idea connected with the collection of baseball cards. If one finds a duplicate, the set must be finite and if at any stage one counts up the number of single different cards $(A)$ and the number of duplicates $(B)$ the size of the set $(n)$ may be estimated from the statistical formula $n=A(A+2 B) / 2 B$. Harwit then applies this to astronomical discoveries of which he considers there are 43 distinct ones, and of these seven are recognized in two different ways. $\mathrm{He}$ is very careful about defining what he means by different ways, that is by totally unrelated 